JYFL 97-11

FIAN/TD-11/97

\title{
Dilepton Emission from Dense Hadron Gas
}

\author{
A.V. Leonidov ${ }^{(1)}$ and P.V. Ruuskanen ${ }^{(2)}$ \\ (1) P.N. Lebedev Physics Institute, Moscow, Russia \\ (2) University of Jyväskylä, Jyväskylä, Finland
}

\begin{abstract}
Using a Hagedorn resonance gas picture and quark-hadron duality we estimate the dilepton emission rate in the vicinity of the QCD deconfinement phase transition. The result is then used to calculate a dilepton spectrum in ultrarelativistic heavy ion collisions. We show that multibody contributions taken into account in the Hagedorn resonance gas approach provide an enhancement of the production rate of massive dileptons as compared to the previously considered sources.
\end{abstract}




\section{Thermal rate from a dense hadron matter}

The dilepton production in high energy heavy ion collisions has drawn considerable attention as a possible signal for the appearance of a quark-gluon plasma [1], see also the reviews [2]. The main problem, however, is to distinguish the signals coming from the normal hadronic phase and from the quark-gluon plasma. To be able to do so one needs reliable models for the dilepton production. At extremely high temperatures the situation is relatively clear. Due to the asymptotic freedom of QCD the dominant production mechanisms are perturbative. At very low temperatures we also have a reliable description of dilepton production in a rarefied hadron gas, predominantly by pion annihilation. Much less can be said about the intermediate temperature range around the phase transition temperature $T_{c} \sim 160 \mathrm{MeV}$, where the hadronic gas is very hot and dense and the usual methods do not work, but the quark-gluon plasma is cold and rarefied and the perturbative QCD results are less reliable. We shall address the problem of estimating the dilepton emission from a hadron gas at these intermediate temperatures. This is of special interest because a hadron gas close to the transition temperature $T_{c}$ could be a dominant feature of the final state produced at the present CERN heavy ion experiments with lead beams.

The new experimental data on dilepton production in heavy ion collisions demonstrate a pronounced excess of produced dileptons over those produced by known hadron sources both in low-mass range [3] and at larger masses [4]. This is a clear motivation to look for additional sources of dileptons not taken into account in the conventional treatment. The excess of small-mass dileptons has been extensively discussed (see, e.g., [5, 6]). More related to our discussion is, however, the excess in the intermediate mass range $1.5 \mathrm{GeV} \leq M \leq 2.5$ $\mathrm{GeV}$ by roughly a factor of 2 with respect to expected contributions from the known sources. This is because the theoretical description we are developing is valid only for sufficiently large invariant masses of lepton pairs. The results will be shown for the mass interval $1.5 \mathrm{GeV}$ $\leq M \leq 4 \mathrm{GeV}$. We shall argue, that by improving the treatment of dilepton emission from the dense and hot hadron gas at the vicinity of $T_{c}$ one can get such an increase with respect to the conventional description.

In [7] we have proposed to use a Hagedorn resonance gas picture and quark-hadron duality to calculate the dilepton emission rate in the hadron phase at temperatures close to the critical one. Below we shall refine these arguments and present additional support to the main conclusion of [0]. In the next section we shall construct an interpolation for the dilepton emission rate satisfying the conditions following from the quark - hadron duality and use it in a hydrodynamical model for heavy ion collisions. In the last section we formulate our 
conclusions.

The problem of calculating the dilepton emission rate in a hadron gas including also the mass range $1.5 \mathrm{GeV} \leq M \leq 4 \mathrm{GeV}$ has been considered in [8] and [9]. We compare the results of [8, 10] for the rate from binary hadron collisions with our results from the dense resonance gas at $T_{c}$. We shall comment on the relation of our results to those of [9]. Since our treatment effectively averages over mass intervals of the order of vector meson widths and mass differences, we cannot address the problem of medium effects on individual resonance parameters, relevant for the low-mass region of CERES results [3].

Let us first remind of the picture of dilepton production at a critical temperature proposed in [7]. The main idea can be formulated as follows. In the vicinity of the critical point the hadronic matter is extremely dense $\mathrm{B}$ and the interactions between the hadrons are very strong. The idea of a Hagedorn resonance gas approach [11] is to encode the infinitely complicated strong interaction physics of a hot and dense hadron matter in a single function, an exponentially rising mass spectrum

$$
\rho(m)=c \frac{1}{m^{a}} e^{m / T_{0}} .
$$

The main idea proposed in [7] was that the exponential spectrum in Eq. (1) takes care of strong interactions only. The free massive degrees of freedom saturating the partition function of the Hagedorn gas can still experience electromagnetic and weak decays. Of a particular interest to us is the spectrum of vector mesons, which are the only important source of dilepton production in the Hagedorn resonance gas in the considered mass range.

In the vector dominance model, to be used below, the dilepton width of a vector meson is given by

$$
\Gamma_{M}^{l+l-}=\frac{1}{g^{2}(M)} \frac{4 \alpha^{2} M}{3} .
$$

The spectrum of dileptons produced by the decays of vector resonances belonging to the Hagedorn gas can be written in the relativistic kinetic theory in the form

$$
\begin{aligned}
& \frac{d R_{V}^{l+l-}}{d M^{2}}=\int d m \rho_{V}(m) \int \frac{d^{3} p}{2 E(2 \pi)^{3}} \exp (-E / T) \int \frac{d^{3} p_{1}}{2 E_{1}(2 \pi)^{3}} \frac{d^{3} p_{2}}{2 E_{2}(2 \pi)^{3}} \\
& \quad \times \overline{\left|\mathcal{M}\left(p \rightarrow p_{1}+p_{2}\right)\right|^{2}}(2 \pi)^{4} \delta^{(4)}\left(p-p_{1}-p_{2}\right) \delta\left(\left(p_{1}+p_{2}\right)^{2}-M^{2}\right),
\end{aligned}
$$

where $p$ is the four-momentum of a decaying vector meson with a mass $m, E=\sqrt{\mathbf{p}^{2}+m^{2}}$ is its energy, $p_{1,2}$ are the four-momenta of the emitted lepton and antilepton, and $\rho_{V}(M)$ is the subspectrum of vector mesons in Eq. (1), not necesserily exponential. In Eq. (3) we have assumed a Boltzmann distribution for the energy of the decaying meson which holds with a very good accuracy provided the invariant mass is high enough. 
In the above formula the squared matrix element for the $V \rightarrow l^{+} l^{-}$decay, including spin summation and averaging, has the form

$$
\overline{\left|\mathcal{M}\left(p \rightarrow p_{1}+p_{2}\right)\right|^{2}}=\left(\frac{4 \pi \alpha}{g(m)}\right)^{2} \frac{4}{3}\left(p_{1}+p_{2}\right)^{2},
$$

which also gives the the result Eq. (2) for the decay width. For the dilepton emission rate one gets from Eq. (3)

$$
\frac{d R_{V}^{l+l-}}{d M^{2}}=\rho_{V}(M) \frac{\alpha^{2}}{6 \pi g^{2}(M)} M^{2} T K_{1}(M / T) .
$$

The most important point is now to fix the mass dependence of the coupling constant $1 / g(M)$. Here we can use the known cross section of $e^{+} e^{-}$annihilation into hadrons which takes place in the same vector channel we are considering. The cross section of $e^{+} e^{-}$annihilation into heavy vector mesons characterized by the mass spectrum $\rho_{V}(M)$ reads:

$$
\sigma\left(e^{+} e^{-} \rightarrow V\right)=\frac{(2 \pi)^{3} \alpha^{2}}{g^{2}(Q)} \frac{1}{Q} \rho_{V}(Q),
$$

where $Q$, the CMS energy of $e^{+} e^{-}$collision, equals the mass of produced vector meson. We proceed by assuming in the spirit of the Hagedorn bootstrap picture that the total cross section of $e^{+} e^{-}$annihilation into hadrons is saturated by the production of vector meson states. This allows us to relate the emission rate from the Hagedorn gas to the total $e^{+} e^{-} \rightarrow$ hadrons cross section. We first have

$$
\sigma\left(e^{+} e^{-} \rightarrow \text { hadrons }\right)=R^{\exp }(Q) \frac{4 \pi}{3} \frac{\alpha^{2}}{Q^{2}}=\sigma\left(e^{+} e^{-} \rightarrow V\right)(Q) .
$$

From the above two equations we get the the mass dependence of the coupling constant

$$
\frac{1}{g^{2}(M)}=\frac{R^{\exp }(M)}{2 \pi^{2}} \frac{1}{M} \frac{1}{\rho_{V}(M)}
$$

and finally, by inserting this into Eq. (4), we can calculate the dilepton production rate in terms of known quantities:

$$
\frac{d R_{V}^{l+l-}}{d M^{2}}=R^{\exp }(M) \frac{\alpha^{2}}{6 \pi^{3}} M T K_{1}(M / T) .
$$

Let us rewrite the function $R^{\exp }(M)$ as

$$
R^{e x p}(M) \equiv R^{\text {part }}+\delta R(M)
$$


where we have decomposed $R^{\exp }(M)$ into a sum of its parton model prediction $R^{\text {part }}=$ $N_{c} \sum_{f} e_{f}^{2}$, where $e_{f}$ is an electric charge of the quark in units of the electron charge, and the deviation from the parton model result, $\delta R(M)$. Experimentally one has $R^{\text {exp }} \sim R^{\text {part }}$ with a good accuracy for the mass-averaged cross section even though the local mass spectrum shows a visible resonance structure parametrized by $\delta R$. From Eqs. (8,9) we get

$$
\frac{d R_{V}^{l+l-}}{d M^{2}}=R^{p a r t} \frac{\alpha^{2}}{6 \pi^{3}} M T K_{1}(M / T)+\delta R(M) \frac{\alpha^{2}}{6 \pi^{3}} M T K_{1}(M / T),
$$

or finally

$$
\frac{d R_{V}^{l+l-}}{d M^{2}}=\frac{d R_{q}^{l+l-}}{d M^{2}}+O(\delta R)
$$

This result shows that the parton model emission rate of dileptons with mass $M$ equals that from the resonance gas at $T \sim T_{c}$ with the same accuracy as parton model describes the total cross section for $e^{+} e^{-} \rightarrow$ hadrons at the CMS energy $M$. We should like to stress, that this result does not depend on a particular assumption on the form of the heavy vector meson spectrum. A natural interpretation of the result is that hadrons as local annihilating degrees of freedom do not provide a simple description of the dilepton production in the immediate vicinity of a critical point, where they start to dissolve to their partonic constituents. As the correlations among the constituents get weaker the electromagnetic current, when probed through the production of heavy lepton pairs, is effectively that of quarks.

This picture leads us in turn to a conjecture on the general behaviour of the dilepton rate as a function of temperature. Let us recall, that a formal nonperturbative expression for the dilepton rate in the hadron gas reads [12], [13]

$$
\frac{d N}{d^{4} q}=-\frac{\alpha^{2}}{6 \pi^{3} q^{2}} \int d^{4} x e^{-i q x}<<J^{\mu}(x) J_{\mu}(0)>_{T}
$$

Basing on Eq. (10) and the above-described physical picture it is tempting to suggest that at high enough dilepton masses the current-current correlator in (11) and thus the dilepton emission rate is to a good accuracy a continuous function of temperature across the phase transition point. How low in the dilepton invariant mass does this hold is determined by the accuracy related to the approximation made in Eq. (6). It would be interesting to check this conjecture in lattice stimulations.

As our aim is to calculate dilepton production in heavy ion collisions, we shall need a continuous rate interpolation from the hadron rate to the QCD one as a function of temperature. A simple way of constructing an interpolation which will agree with Eq. (10) is to start from the rate due to binary hadron collisions calculated in 8 and match it to the QCD one at the critical temperature $T_{c}$. 
Before turning to the actual hydrodynamic calculation, let us also mention a calculation in [9] based on using the soft pion theorems in calculating the hadronic contribution to the polarization tensor. The main result of this paper for thermal dilepton rate from the hadron gas can be rewritten in the form

$$
\frac{d R_{(s) h}^{l+l-}}{d M^{2}}=\frac{d R_{q}^{l+l-}}{d M^{2}}\left[1-\left(\varepsilon-\frac{\varepsilon^{2}}{2}\right)\left(\frac{\rho^{V}(M)-\rho^{A}(M)}{\rho^{e m}(M)}\right)\right]\left(1+\delta R / R^{\text {part }}\right),
$$

where $\varepsilon=T^{2} / 6 F_{\pi}^{2}, F_{\pi}=93 \mathrm{MeV}$ and $\rho^{e m}, \rho^{V}$ and $\rho^{A}$ are the suitably defined electromagnetic, vector and axial spectral densities which can be extracted from measured quantities (for details see [9]). In the following we shall be interested in the production of dilepton pairs having invariant masses $M>1.5 \mathrm{GeV}$. In this mass range one can neglect $\rho^{A}$ with respect to $\rho^{V}$ and take $\rho^{V}=\rho^{e m}[9]$. This gives, when neglecting $\delta R$,

$$
\frac{d R_{(s) h}^{l+l-}}{d M^{2}}=\frac{d R_{q}^{l+l-}}{d M^{2}}\left[1-\left(\varepsilon-\frac{\varepsilon^{2}}{2}\right)\right] .
$$

It is interesting that the temperature dependence through $\varepsilon$ is in fact quite smooth, so the rate is predicted to be close to the thermal quark-antiquark one even at temperatures below $T_{c}$. This obviously agrees with our main result Eq. (10). Unfortunately the range of masses where experimental information is available for the technique of [9] is limited to $M \leq 2.2$ $\mathrm{GeV}$, so to analyze a broader mass interval one in forced to use a more phenomenological approach as described below.

Let us now turn to the calculation of the dilepton emission rate from a hadron gas due to binary collisions of mesons [8]. In view of the previous discussion it is of special interest and importance to look how close is the rate of this approach to our result. This will provide valuable information on the relative importance of the higher resonances and the multibody effects in dilepton emission processes. In the Boltzmann approximation for the thermal distribution, which works fairly well when the invariant masses of lepton pairs satisfy $M>1.5 \mathrm{GeV}$, the rate reads

$$
\frac{d R_{(b) h}^{l+l-}}{d M^{2}}=\alpha^{2} \frac{\sigma_{h}(M)}{(2 \pi)^{4}} M T K_{1}(M / T),
$$

where $\sigma_{h}(M)$ is calculated from the specific Lagrangian for meson interactions [8]. In the following we shall use the rate Eq. (14) including the following reactions (for brevity we list only the incoming particles) [10]: $\pi^{+} \pi^{-}, K^{+} K^{-}, K^{0} \bar{K}^{0}, \rho^{+} \rho^{-}, K^{*+} K^{*-}, K^{* 0} \bar{K}^{* 0}, \pi \rho, \pi^{0} \omega$, $\pi^{0} \phi, \eta \rho^{0}, \eta \omega, \eta \phi, \eta^{\prime} \rho^{0}, \eta^{\prime} \omega, \eta^{\prime} \phi, K^{+} K^{*-}+K^{-} K^{*+}, K^{0} \bar{K}^{* 0}+\bar{K}^{0} K^{* 0}, \pi^{0} \omega(1420), \pi^{0} \omega(1660)$.

\footnotetext{
${ }^{1}$ We are very grateful to P. Lichard for providing us the numerical data for this rate.
} 


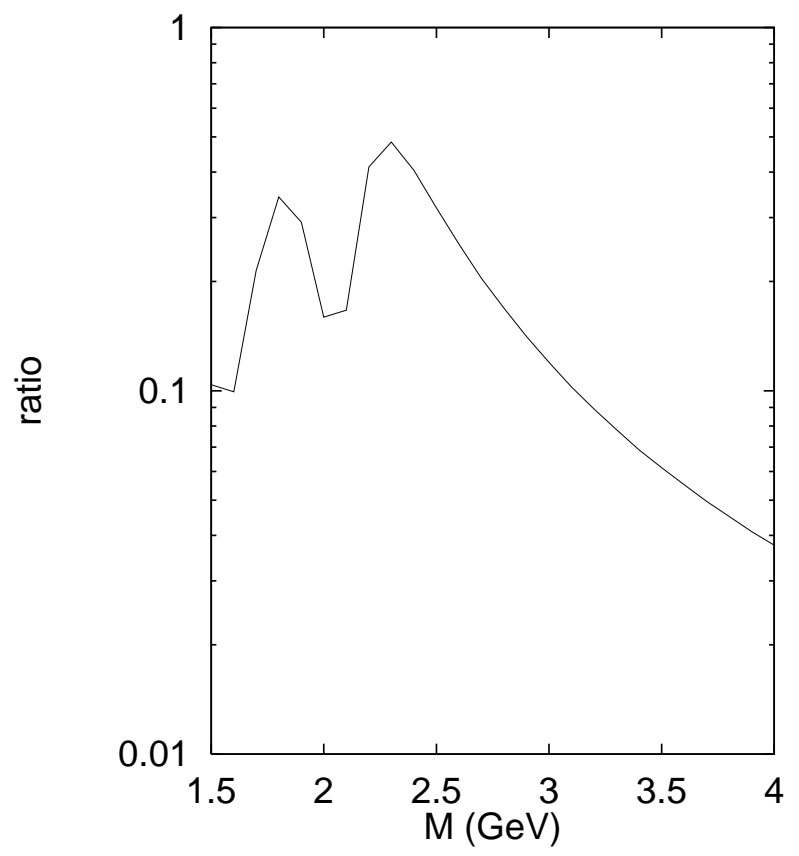

Figure 1: Ratio of static hadronic dilepton production rate in a binary collision approximation to the quark-antiquark one at $\mathrm{T}=160 \mathrm{MeV}$

In Fig. 11 we show the ratio of this binary hadron rate to our result Eqs. (8) and (10) at $T=160 \mathrm{MeV}$ in the mass interval of interest. We see that the rate from binary interactions is less than our rate which takes into account also higher resonances and multibody effects and, in the approximation we use, equals the rate from quark-antiquark annihilations at this temperature. In the Hagedorn resonance gas approach the critical temperature is expected to be of order $\left(T_{c} \sim 160 \mathrm{MeV}\right)$, so taking into account contributions from binary collisions only is not sufficient at least in the vicinity of the critical point. In the end of the next section we shall discuss the numerical importance of this in calculating dilepton production in heavy ion collision in a hydrodynamic model, but already now one could notice, that the dependence on temperature in the above ratio cancels (cf. Eqs. (8) and (14), so the difference is directly translated into the changes in the total dilepton rate.

\section{Dilepton emission in a hydrodynamic model}

In this section we shall study the implications of the above considerations on dilepton production in heavy ion collisions described with the simplest one-dimensional scaling hydrodynamic model [15]. The resulting dilepton rate is obtained by integrating over the space-time 
history of the expansion.

The main result of the previous section is the approximate continuity of the thermal emission rate of massive lepton pairs across the phase transition point. Thus the expression for the thermal dilepton emission in hadron phase used in hydrodynamical calculations should be constructed to ensure this continuity matching. In the narrow mass range, where the experimental data on spectral densities is available one can apply Eq. (12) with the approximate continuity automatically satisfied. To describe the dilepton mass spectrum in a wider mass range, as needed to compare with experiment, one needs effective approaches like that in [8]. Since the binary reactions should be the main source of dileptons at low densities and temperatures and since we expect our results to apply best in the vicinity of the critical temperature, we will construct an interpolation procedure which at $T_{c}$ equals the quark matter rate and goes over to the binary hadron rate of [8] a low temperatures.

Below we shall consider the cases of first order and second order deconfinement phase transition. The critical temperature in both cases is chosen to be $160 \mathrm{MeV}$ and the freezeout temperature $130 \mathrm{MeV}$. The basic difference between these two cases is the presence or absence of a mixed phase. From the arguments of the previous section it is clear, that for the dilepton emission the rate should stay equal to the quark-antiquark one for the whole duration of mixed phase.

Let us rewrite a well-known basic formula for the spectrum of dileptons emitted during the one-dimensional scaling expansion (see e.g. [14], [16]) in the form

$$
\frac{d N^{l+l-}}{d M d y}=\pi R_{A}^{2} \int_{\tau_{1}}^{\tau_{2}} d \tau \tau r_{q(h)}^{q(m, h)}(T(\tau)) \frac{d R_{q(h)}^{l+l-}}{d M^{2}}(M, T(\tau)),
$$

where $R_{A}$ is the radius of the smaller of the two colliding nuclei, $r_{q, h}^{q(m, h)}$ specifies the weight for the quark matter and hadron gas thermal rates (lower index) in each of the phases, quark matter, mixed or hadron gas (upper index). A head-on collision is assumed. To specify completely the evolution of the system one has to know the equations of state for both phases and the nature of the phase transition. The entropy of the quark-gluon phase will be taken equal to that of the ideal gas of gluons and three lightest quarks

$$
S_{q}(T)=\frac{74 \pi^{2}}{45} T^{3}+\frac{1}{\pi^{2}}\left(12 m_{s}^{2} T K_{2}\left(m_{s} / T\right)+3 m_{s}^{3} K_{1}\left(m_{s} / T\right)\right) .
$$

A hadron phase will be described by a free resonance gas including all particles with the masses up to $2 \mathrm{GeV}$. The dependence of temperature on proper time $T(\tau)$ follows from the Bjorken equation 15

$$
S(T(\tau))=\frac{1}{\tau} \frac{3.6}{\pi R_{A}^{2}} \frac{d N}{d y}
$$


Let us start with the case when the deconfinement transition from the hadron gas to the quark-gluon plasma is of second order. Here the entropy is continuous across the phase transition point $T_{c}$. We shall assume that at all temperatures the entropy is given by a slightly generalized form of an interpolation used in [17])

$$
S(T)=(1-\eta(T)) S_{h}(T)+\eta(T) S_{q}(T)
$$

where

$$
\eta(T)=\frac{1}{2}\left(1+\tanh \left[\left(T-T_{c}\right) / \Delta T\right]\right)
$$

and $\Delta T$ specifies the width of the transient zone where the entropy density of one phase goes over to that of the other phase. We shall take $\Delta T=16 \mathrm{MeV}, 10 \%$ of $T_{c}$. Let us note, that the interpolation (18) does not imply that the phases are mixed at any temperature, it just provides a smooth interpolation between the two known asymptotic expressions. The dilepton emission rate is equal to that from quark matter, $d R_{q}^{l+l-} / d M^{2}$, above $T_{c}$, i.e. the corresponding weights in Eq. (15) at $T>T_{c}$ are $r_{h}^{q}=0$ and $r_{q}^{q}=1$. Below $T_{c}$ it is chosen to be a combination of the quark matter and hadronic rates:

$$
\left.\frac{d R^{l+l-}}{d M^{2}}\right|_{T<T_{c}}=r_{q}^{h}(T) \frac{d R_{q}^{l+l-}}{d M^{2}}+r_{h}^{h}(T) \frac{d R_{h}^{l+l-}}{d M^{2}} .
$$

The weights in this equation are

$$
\left\{\begin{aligned}
r_{q}^{h}(T) & =\eta(T) /(1-\eta(T)), \\
r_{h}^{h}(T) & =1-r_{q}(T)
\end{aligned}\right.
$$

ensuring that at $T=T_{c}$ the overall rate equals that from the quark matter and providing an interpolation between the quark rate at $T_{c}$ and hadron rate at low temperatures. As discussed in detailed in the previous section, the necessity of an extra contribution in the hadronic phase is due to the necessity of taking into account the effects beyond the binary collision approximation, in which the results of [8] were obtained. Otherwise Eq. (10) will not hold at $T=T_{c}$.

In Fig. 2 we show the dilepton spectra calculated for two different particle densities corresponding to head-on $\mathrm{Pb}-\mathrm{Pb}$ collisions at zero rapidity at $\mathrm{SPS}(d N / d y=600$, lower curves) and RHIC ( $d N / d y=1500$, upper curves) energies. The dashed curves correspond to the binary collision approximation to the hadronic dilepton rate and the solid ones to the rate interpolation Eq. (20). We see that at SPS energies the contribution from the extra sources in hadron phase which are not taken into account in the binary collision approximation with a limited number of interacting mesons is visible in the resulting dilepton spectrum 


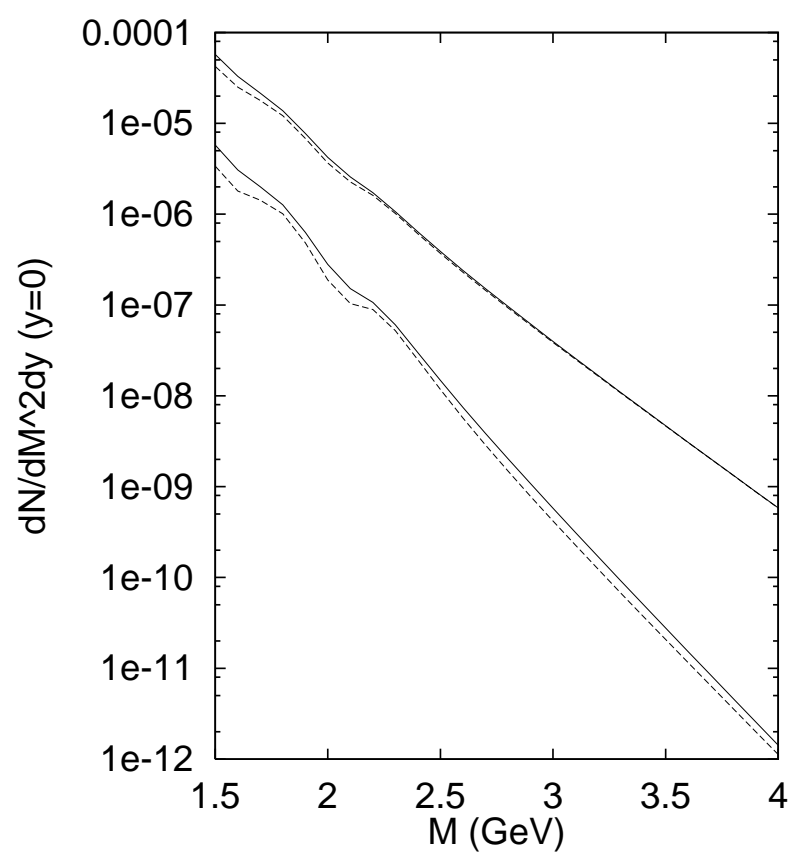

Figure 2: Dilepton spectra for SPS (lower curves) and RHIC (upper curves) for second order phase transition. The dashed curves correspond to a binary collision approximation to the hadronic dilepton rate, the solid ones correspond to the rate interpolation Eq. (20).

and brings a multiplicative factor of the order of 1.5. At RHIC energies the signal is already almost totally dominated by pairs from quark-antiquark annihilations at the hot early stages of the collision $\left(T \geq T_{c}\right)$. Thus the details of the description of the hadronic electromagnetic currents in this mass range, even in the vicinity of $T_{c}$, are no longer important.

Let us now consider the case of a first order phase transition. The basic difference from the previous case is that the matter evolves from the quark phase to the hadron one through a mixed phase. Since in our approach at $T=T_{c}$ the rate in hadron phase approximately equals that in quark phase, the weights in the mixed phase are $r_{q}^{m}=1$ and $r_{h}^{m}=0$. This enhances the contribution from the mixed phase as compared to the standard scenario in which only binary hadron collisions are producing dileptons from a volume occupied by hadronic phase throughout the time the mixed phase exists (cf. Fig. 1). In the hadronic phase, $T<T_{c}$, the rate is taken to be the same superposition of quark and hadron rates, Eqs. (20) and (21), as in the case of second order transition. The results of calculation for $\mathrm{Pb}-\mathrm{Pb}$ collisions at SPS and RHIC energies are plotted in Fig. 3. Here the picture is somewhat more complicated than in the case of second order transition. The main conclusions can be formulated as follows. At SPS energies the system is created at a temperature close to a critical one, 


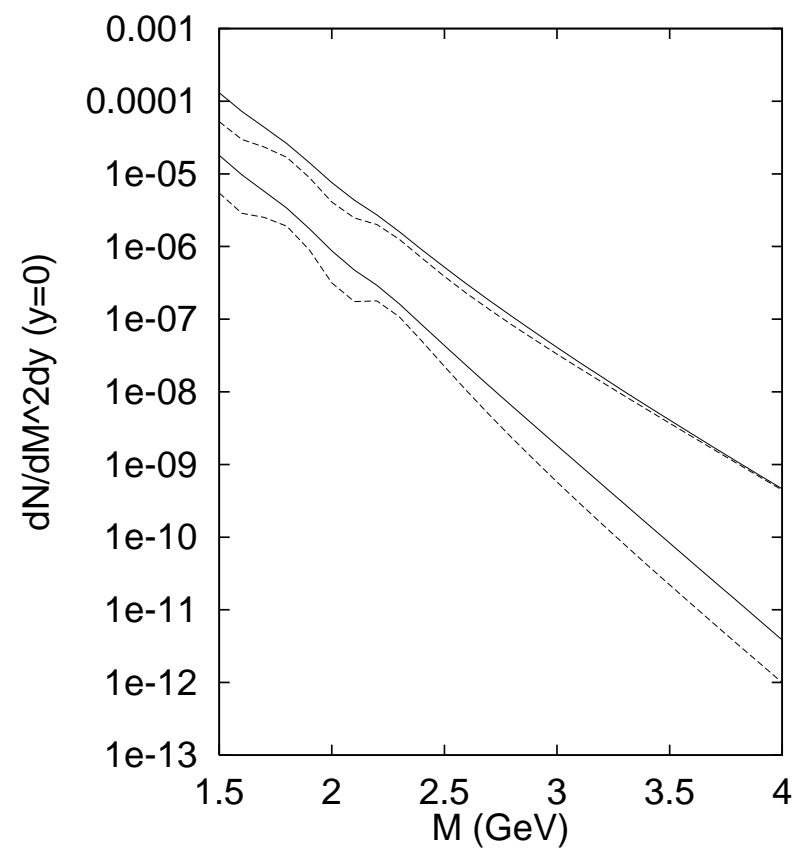

Figure 3: Dilepton spectra for SPS (dashed line) and RHIC (solid line) for the first order phase transition.

so the main contribution is coming from the mixed and low-temperature phases. The new sources enhance the dilepton rate approximately with a factor of 3 - 4 in the mass range under consideration. The contributions originating from the new sources in the mixed and the low-temperature phase are of the same order. At RHIC energies the contribution of the high temperature phase is already quite significant at $M=2 \mathrm{GeV}$ and dominates at $M=4$ $\mathrm{GeV}$. The new sources enhance the rate with a factor 2 at $M \sim 2 \mathrm{Gev}$ and are no longer significant at $M \sim 4 \mathrm{GeV}$.

Let us note, that our main goal in this model calculation is to get a relative enhancement of the dilepton rate as compared to the usual calculations exploiting binary collision approximation. A well-known drawback of the one-dimensional scaling expansion are the long expansion times, $\sim 100 \mathrm{fm}$ already at SPS energies. In a more realistic three-dimensional treatment the relative time scales for different stages of system's evolution can change, but the overall relative enhancement for the contribution from the hadron phase can be expected to be more stable. In particular, this should be the case at SPS where the mixed and hadron phases are expected to dominate. 


\section{Conclusion}

We have presented a method for taking into account the higher order virial corrections to the dilepton emission rate in a hot and dense hadron gas. To go beyond a binary collision approximation with a limited number of interacting meson species we argue that the thermal emission rates of large mass lepton pairs from the quark matter and dense hadron gas are approximately equal in the vicinity of $T_{c}$. We then construct an interpolation between the hadron rate due to binary collisions at low $T$ and the rate at the critical point $T=$ $T_{c}$, effectively the quark matter rate. The resulting rates are used in a one-dimensional hydrodynamical description of heavy ion collisions at SPS and RHIC energies. The main result in the case of a second order deconfinement transition is that while at SPS energies the higher order virial corrections to the dilepton rate from the binary hadron collisions are still seen in the total thermal dilepton spectrum, at RHIC energies the emission from quark matter above $T_{c}$ dominates in the considered mass range $1.5 \mathrm{GeV} \leq M \leq 4 \mathrm{GeV}$. In the case of a first order deconfinement phase transition the rate enhancement due to new sources is bigger than in the second order case at SPS energies. At RHIC it can still be seen at the low-mass end of the mass interval under consideration $(M \sim 2 \mathrm{GeV})$ but is no longer important at $M=4 \mathrm{GeV}$, where the emission from the high temperature QGP phase becomes dominant.

A. Leonidov acknowledges the warm hospitality at the University of Jyväskylä, where a major part of this work was done. His work was partially supported by the Russian Foundation for Basic Research under grant 93-02-3815 and by the Academy of Finland under grant 27574 .

\section{References}

[1] E.L. Feinberg, Nuovo Cim. A34 (1976) 391; E.V. Shuryak, Phys. Lett. B78 (1978) 150; G. Domokos and J.I. Goldman, Phys. Rev. D23 (1981) 203.

[2] P.V. Ruuskanen, Nucl. Phys A544 (1992) 169c; J.I. Kapusta, Nucl. Phys. A566 (1994) $45 \mathrm{c}$.

[3] G. Agakichev et al., NA45 Collaboration, Nucl. Phys. A610 (1996), 317c

[4] M.C. Albreu et al., NA50 Collaboration, Nucl. Phys. A610 (1996), 331c

[5] R. Baier, M. Dirks and K. Redlich, hep-ph/9612448 
[6] C.Q. Li, C.M. Ko and G.E. Brown, Nucl. Phys. A606 (1996), 568; C.M. Hung and E. Shuryak, hep-ph/9608299

[7] A.V. Leonidov and P.V. Ruuskanen, Heavy Ion Phys. 1 (1995), 61

[8] C. Gale, P. Lichard, Phys. Rev. D49 (1994), 3338

[9] Z. Huang, Phys. Lett. B361 (1995), 131

[10] P. Lichard, Private communication.

[11] R. Hagedorn, Nouvo Cim. Suppl. 3 (1965), 117

[12] L.D. McLerran and T. Toimela, Phys. Rev. D31 (1985), 545,

[13] H.A. Weldon, Phys. Rev. D42 (1990), 2384,

[14] K. Kajantie, J. Kapusta, L. McLerran and A. Mekjian, Phys. Rev. D34 (1988), 2746

[15] J. Bjorken, Phys. Rev. D27 (1983), 140

[16] P.V. Ruuskanen, in Quark - Gluon Plasma, ed. by R. Hwa, World Scientific, 1986, p. 519

[17] J.-P. Blaizot and J.-Y. Ollitraut, in Quark-Gluon Plasma, ed. by R. Hwa, World Scientific, 1986, p. 393 